\title{
Clinical Efficacy Analysis of Using the Five Bodies to Treat Knee Degenerative Joint Disease
}

\author{
Jing Xie ${ }^{1, a}$, Dongli Jin ${ }^{1, b}$, Dongliang Shi, c \\ ${ }^{1}$ Henan Province Hospital of Traditional Chinese Medicine, Zhengzhou, Henan, 450002 \\ a email, ${ }^{\mathrm{b}}$ email, ${ }^{\mathrm{c}}$ email
}

Keywords: Five Bodies Method, Knee Degenerative Joint Disease, Treatment

\begin{abstract}
Objective: To observe and analyze the clinical efficacy of using five bodies pass notes to treat knee degenerative joint disease. Methods; The study is from March 2015 to March 2016 in our hospital for treatment of knee degenerative joint disease patients, 80 cases were randomly divided into odd and even method in the control group ( $\mathrm{n}=40$ patients) and observation group ( $\mathrm{n}=40$ patients). By using through-line method transfer five body massage and acupuncture and Chinese Herbs plus extubation law giving patients in the observation group, the use of oral ibuprofen and glucosamine sulfate treatment administered to the control group. Comparative efficacy of two methods of treatment and pre-treatment and post-treatment after the first course and second course of joint function score. Results; after the first and second courses, excellent rate of patient observation crude rate than the control group, the difference was significant, statistically significant, $\mathrm{p}<0.05$. After treatment, the two groups of patients HSS scores were increased, the difference was statistically significant, $\mathrm{p}<0.05$; observation group after treatment score after the first course of treatment and the second treatment scores were higher, the difference was statistically significant, $\mathrm{p}$ $<0.05$. Conclusion; knee degenerative joint disease through using the five-body transfer method of treatment, the effect is significant.
\end{abstract}

\section{Introduction}

Knee degenerative joint disease is also known as osteoarthritis of the knee in clinical medicine, clinically multiple, common symmetry joint disease. The main clinical manifestations of knee and knee pain, and restricted hand knee stiffness and so on. In the clinical treatment of the use of Chinese medicine treatment methods are varied, but the effect is unsatisfactory, this study used five body Therapy pass notes, and get a better therapeutic effect, are reported below.

\section{Subjects and Methods}

The study of March 2015 March 2016 in our hospital for treatment of knee degenerative joint disease patients, 80 cases in this study the relevant literature in diagnostic criteria, all subjects using imaging and pathological diagnosis, combined with signs and clinical manifestations of knee diagnosed as degenerative joint disease. The control group of patients, 27 females, 13 males; the minimum age is 48 years old and the oldest was 78 years, median age $(59.4 \pm 7.1)$ years; duration (21.1 \pm 7.5$)$ years; the observation group patients, 26 women cases, male 14 cases; the minimum age is 52 years old, the maximum age is 79 years, with a median age (59.8 \pm 8.1 ) years; duration $(22.1 \pm 8.5)$ years; all subjects for age, sex, duration and other baseline data, by using SPSS19.0 statistical software package for data processing, the results showed no statistically significant, comparable, $\mathrm{p}>0.05$.

By using through-line method transfer five body massage and acupuncture and Chinese Herbs plus extubation law giving patients in the observation group, as follows: firstly acupuncture, massage and cupping then, while taking the medicine, each morning and evening meal times. Acupuncture; select points as follows, select Hedingshan affected, the Commission, the inner knee eye, three-legged, the outer knee eye, Cheng-shan, post puncture needle for 30 minutes. Acupuncture treatment administered to a patient once a day. Cupping; according to the 
above-mentioned points selecting the right size of cupping, administered to a patient cupping, leaving cans of time not too long, avoid blisters due to the long retention time appeared, 5 minutes, once a day. Massage; first patient supine, the surgical staff from the position of the greater trochanter in patients with ipsilateral femur, from the gallbladder through row after Qiuxu Point rubbing method, a total of six times. Then supine position, the surgical staff from the position of the greater trochanter in patients with ipsilateral femur, from the gallbladder through row after Qiuxu Point rubbing method, and the main point of rubbing: absolutely bone, the wind market, Qiu Hui, Yanglingquan 4 points, a total of three times. Position the patient supine knees, rubbing the ipsilateral yinlingquan, a sea of blood, Liang Qiu, outside the knee eyes Zusanli, crane top, inner knee eye and other points, 10 times each. Then the surgeon single-handedly holding the distal end of the patient's leg, and the other hand to hold back the patient's patella, with the thumb and four fingers hold the additional pain points around the joint, giving the patient the joint adduction and external rotation and abduction internal rotation of cranking. Patients were prone position, there Cheng Fu points to the bladder through the loop and then administered to the patient to the Achilles tendon rubbing method, a total of three times, once a day. Traditional Chinese medicine; administered to the patient and leg pain soup Juan were treated by the prescription: Salvia, Eupolyphaga, parasitic, centipedes, Sichuan off, Woodwardia, Aconitum, sea breeze rattan, Aconitum, Caulis, look for bone wind three seven, licorice, at thirty-seven, the other drug fried twice, juice and PNG powder blunt, sooner or later, the 1st. 10 days for a course of treatment, continuous treatment for 20 days. Oral ibuprofen (at a dose of $600 \mathrm{mg}, 3$ times a day) and glucosamine sulfate (at a dose of 500mg, 3 times a day) administered in the control group. 10 days for a course of treatment, continuous treatment for 20 days.

All subjects during treatment with resting mainly to avoid weight-bearing knee, disable all associated with the disease of Western medicine. At the same time after the first course of treatment and the first two courses evaluated.

According to the relevant literature in the scoring standards knee pain, pain 30 points, 22 points and recovery of joint function, deformity 10 points, 18 points of motion, muscle strength 10 points, a total of 100 points. Good 60 to 79 minutes, is less than 69 points. Excellent good + = good rate.

The collected set of experimental data, statistical software for data processing in data package SPSS20.0, count data and measurement data were applied (n\%) and ( $\square \mathrm{x} \pm \mathrm{s}$ ) that the differences between the groups by $\mathrm{x}^{2}$ and t-test, when $\mathrm{P}<0.05$, the difference was statistically significant.

\section{Results}

Comparison of the Efficacy of the Two Groups after the Treatment. After the first and second courses, excellent rate of patient observation crude rate than the control group, the difference was significant, statistically significant, $\mathrm{p}<0.05$. See Table 2 .

Table 1 After the end of the course 1 were compared efficacy [n (\%)]

\begin{tabular}{lllll}
\hline Group & Number of cases & Good $(\mathrm{n})$ & Ok $(\mathrm{n})$ & NG $(\mathrm{n})$ \\
\hline Observe Gr. & 40 & $15(37.50)$ & $21(52.50)$ & $4(10.00)$ \\
Competition Gr. & 40 & $7(17.50)$ & $13(32.50)$ & $20(50.00)$ \\
$\mathrm{X}^{2}$ & & 10.0313 & 8.1841 & 38.0952 \\
$\mathrm{p}$ & & 0.0015 & 0.0042 & 0.0000 \\
\hline
\end{tabular}


Table 2 After the first two courses in Table 2 were compared efficacy (Example [n (\%)]

\begin{tabular}{lllll}
\hline Group & Number of cases & Good $(\mathrm{n})$ & Ok $(\mathrm{n})$ & NG $(\mathrm{n})$ \\
\hline Observe Gr. & 40 & $22(55.00)$ & $16(40.00)$ & $2(5.00)$ \\
Competition Gr. & 40 & $16(40.00)$ & $14(35.00)$ & $10(25.00)$ \\
$\mathrm{X}^{2}$ & & 4.5113 & 0.5333 & 15.6863 \\
$\mathrm{p}$ & & 0.0337 & 0.4652 & 0.0000
\end{tabular}

Before Treatment, HSS Scores Comparison after the First and Second Treatment. After treatment, the two groups of patients HSS scores were increased, the difference was statistically significant, $\mathrm{p}<0.05$; observation group after treatment score after the first course of treatment and the second treatment scores were higher, the difference was statistically significant, $\mathrm{p}<0.05$. See Table 3.

Table 3 before treatment, after the end of the first and second treatment HSS scores ( $\square \mathrm{x} \pm \mathrm{s}$ )

\begin{tabular}{lcccc}
\hline Group & cases & Before Treat & 1 Treatment & 2 Treatment \\
\hline Observe Gr. & 40 & $61.3 \pm 4.3$ & $77.3 \pm 4.6$ & $83.3 \pm 4.3$ \\
Competition Gr. & 40 & $61.6 \pm 4.5$ & $68.4 \pm 7.9$ & $72.5 \pm 7.4$ \\
$\mathrm{t}$ & & 0.3048 & 6.1574 & 7.9809 \\
$\mathrm{p}$ & 0.7613 & 0.0000 & 0.0000 \\
\end{tabular}

\section{Discussion}

Knee degenerative joint disease is the medicine of Gubi category. When the body upright sparse distribution barriers and lack of righteousness, the evils can enter through the skin reentry tendons, bone, blood circulation can effectively lead to the arrest by the evil place, and thus the occurrence of organ dysfunction and pain phenomenon. So rib portion, the sheath, and the ubiquitous meat portion of the pulse portion while being involved in the middle of the body of clinical symptoms and clinical manifestations very prominent. Since the barriers around the knee revascularization occurred, leading to changes in skin temperature around the knee; at the same time around the knee ligaments and bursa occur inflammatory conditions and so on. So the disease pathological changes in terms of the five bodies with the more common symptoms of the disease.

Knee degenerative joint disease is the virtual real standard of the disease, while five bodies with disease symptoms are more common, so the most suitable five bodies and pass notes and carrot treatment in the clinical treatment. The so-called stasis passes through by means of the five bodies; refers to the so-called adjusted by adjusting the yin and yang of the liver and kidney and bone errors effectively regulate the gas. Late for an efficient use of energy pipelines in smooth and effective at the same time remove saprophytic new, we need to pay close attention around the knee and adjust coordination among organizations. Easy correction gap medial and lateral deformation, eliminate joint soft tissue adhesions, thus effectively improve the range of motion.

In short, through using five-body transfer method to treat the knee degenerative joint disease, the effect is significant.

\section{References}

[1] Wang Yan, Wei Yongge, Hu Xiaozhou. 10- HCPT treatment of mouse collagen-induced arthritis [J] Shandong Traditional Chinese Medicine, 2013, 53 (11): 29-31. 
[2] Liu Dan, Chen Qingping, Zhou Yani. Complement system and other immunomodulatory effects of rheumatoid arthritis [J]. Chinese Journal of Rheumatology, 2014, 18 (3): 204-206.

[3] Teng Hongli, Hu Yuping, Du Guangzhong. Standard research technique Zhuang medicine medicated thread moxibustion treatment of rheumatoid arthritis [J] External Therapy Journal, 2014, 23 (3): 3-5.

[4] Li Manyi, Zhang Ziyang, Lou Yuqian. The isosceles paralysis of Origin and relevant historical literature review (lower) [J] rheumatism and arthritis, 2016, 5 (6): 51-59,76.

[5] Bao Lei, Zhang Huimin, Min Jiayu. Countries to treat gout medicine master experience [J] Global Chinese Medicine, 2016, 9 (5): 610-612. 